\title{
High-fidelity Aeroelastic Analysis of Very Flexible Aircraft
}

\author{
Benjamin P. Hallissy, Carlos E.S. Cesnik ${ }^{\dagger}$ \\ University of Michigan, Ann Arbor, MI 48109
}

\begin{abstract}
This paper describes the development and capabilities of a high-fidelity aeroelastic simulation tool for very flexible aircraft (HiFi-VFA) within a Multi-Disciplinary Computing Environment (MDICE). The code loosely couples a geometrically nonlinear, quasi-3D structural solver with an Euler/Navier-Stokes flow solver capable of arbitrary, large, mesh deformation. Verification of the static aeroelastic solver is presented for a hypothetical high aspect ratio wing discussed in the literature. Time domain aeroelastic simulations using the new code generally agree well with the response of an in-house aeroelastic simulation toolbox, but the high-fidelity results suggest the presence of higher aerodynamic damping than predicted by the finite state aerodynamics. With these results, flutter boundaries are sought using two flutter prediction methods (ARMA and FMDS) and the advantages/disadvantages of these prediction methods in computational aeroelasticity are assessed.
\end{abstract}

\section{Introduction}

$\mathrm{H}^{\prime}$ IGH altitude, long endurance (HALE) aircraft offer capabilities found nowhere else in the spectrum of machine flight, but their design and analysis offers unique challenges. From their observation altitude above 50,000 ft, HALE systems can relay telecommunication signals, perform intelligence, surveillance, and reconnaissance (ISR) functions covering large swaths of land for extended periods of time, and collect valuable data for atmospheric research. ISR capabilities form the primary mission for SensorCraft platforms - designed for at least 30 hours of on-station endurance at a range of 2000 nautical miles [1]. NASA's ERAST [2] program gave rise to a family of all-wing aircraft designed with science missions in mind, which set multiple records for endurance and sustained high-altitude flight. DARPA's Vulture [3] program pushes the endurance envelope farther, aiming to demonstrate an aircraft that can loiter for 5 years, between 60,000 and 90,000 ft, with a $1000 \mathrm{lb}$ payload. Aircraft meeting these performance requirements have characteristic design traits: (1) slender, high aspect ratio wings with thick airfoils, and (2) very low structural weight. Long, lightweight wings tend be very flexible, and even in trimmed flight, HALE aircraft are often deformed beyond the range in which small displacements can be assumed. At high altitude, the speed of sound is low relative to sea level, thus the Mach regime is higher. Since thick airfoils have a lower critical Mach number, these higher Mach flows can generate highly nonlinear aerodynamics in the form of shocks along the wing upper surface.

Numerous researchers have tackled the challenges of HALE aeroelasticity. Van Schoor and von Flotow [4] were among the first who studied this problem. Using linear finite element analysis (FEA) and 2D unsteady strip theory aerodynamics, they pointed out that including flexible deformation modes has a dramatic effect on predicted aircraft stability. Several other studies on HALE wings have used low-order aerodynamics [5-14]. First, Patil, Hodges, and Cesnik authored a series of works [5, 6, 8] about the coupling of an exact intrinsic beam model with Peters' finite-state aerodynamics [15] and its application to

${ }^{*}$ Graduate Research Assistant, Department of Aerospace Engineering, AIAA Member, hallissy@umich . edu

${ }^{\dagger}$ Professor, Department of Aerospace Engineering, AIAA Associate Fellow, cesnik@umich . edu 
highly flexible aircraft. In a parallel effort, Drela [7] developed ASWING, an integrated design and analysis package which incorporated a nonlinear beam model with lifting-line aerodynamics. Patil and Hodges [9] use nonlinear beam theory, with non-planar doublet and vortex lattice aerodynamics for similar applications. Tang and Dowell [10] also incorporate a geometrically nonlinear beam model, but use 2D strip theory for aerodynamics. A comprehensive Nonlinear Aeroelastic Simulation Toolbox (UM/NAST) [11] which uses a strain-based structural formulation and Peters finite-state aerodynamics [15] was developed at the University of Michigan. UM/NAST has since been used to investigate the aeroelastic responses of flying wings [12], blended-wing-body configurations [13], and flapping wing micro air vehicles [16]. Recently, Wang et al. [14] coupled a nonlinear beam model with an unsteady vortex-lattice model (UVLM) which models a free wake and captures non-planar effects and viscous effects (through vortex dynamics). These models, although computationally efficient and sufficiently accurate for design, fall short of computational fluid dynamics (CFD) in terms of accuracy and ability to capture a majority of flow nonlinearities.

Concurrently, researchers have coupled higher-fidelity CFD with beam models. Garcia et al. [17] studied the the effects of transonic aerodynamics by pairing a Navier-Stokes solver (ENSAERO-WING) with a nonlinear beam model, and Smith et al. [18] coupled a structured Euler (ENS3DAE) solver with a geometrically exact beam model to investigate the effect of nonlinear aerodynamics and structures compared to various linear solutions. They concluded that linear aerodynamics theories result in larger steady state displacements and conservative flutter predictions. Palacios and Cesnik [19] coupled a nonlinear, quasi-3D structural solver with ENS3DAE to investigate static aeroelasticity of HALE wings in compressible flow. The camber deformation degree of freedom captured by the structural model in that study dramatically affected the shock position on top of the wing, suggesting inclusion of this deformation mode is critical in any transonic dynamic aeroelastic analysis. Garcia [20] applied nonlinear beam FEA and the thin-layer Navier-Stokes equations to swept and unswept high aspect ratio wings, finding that slight rearward sweep increased the wings susceptibility to tip stall, and that nonlinear, transonic aerodynamics exacerbated this problem. Moving away from beam models, Seber and Bendiksen [21] coupled nonlinear plate FEA with an Euler solver and highlight the importance of in-plane motion on transonic aeroelasticity with moderate to large deflections

The present study extends the work of Palacios and Cesnik [19]. It introduces an unstructured CFD code coupled with a quasi-3D, slender structure model. We then use this capability to investigate static and dynamic aeroelasticity of flexible, high aspect ratio, wings and HALE aircraft in compressible flow.

\section{Methodology}

For this work, we loosely couple a geometrically nonlinear structural solver with an established CFD code and mesh deformation algorithm. This coupling methodology solves the fluid and structural equations in separate modules, and the modules exchange information at each global time step. A Multi-Disciplinary Computing Environment (MDICE) [22], developed by CFDRC under contract with the Air Force, facilitates this exchange. Figure 1 gives a high-level overview of the present coupling procedure.

\section{A. Structure}

A high-fidelity model of the structure is obtained by a mathematically-rigorous process of asymptotic reduction of the general anisotropic slender solid [23,24]. The asymptotic solution method splits the problem into i) a linear analysis over the cross section (Variational-Asymptotic Beam Sectional Analysis - UM/VABS) [24] in which a finite-element procedure solves the cross-sectional displacement and electric field to minimize the local energy state; and ii) a long-scale problem with a kinematically-exact one-dimensional formulation of the dynamics of the reduced structure (Nonlinear Active Beam Solver - 


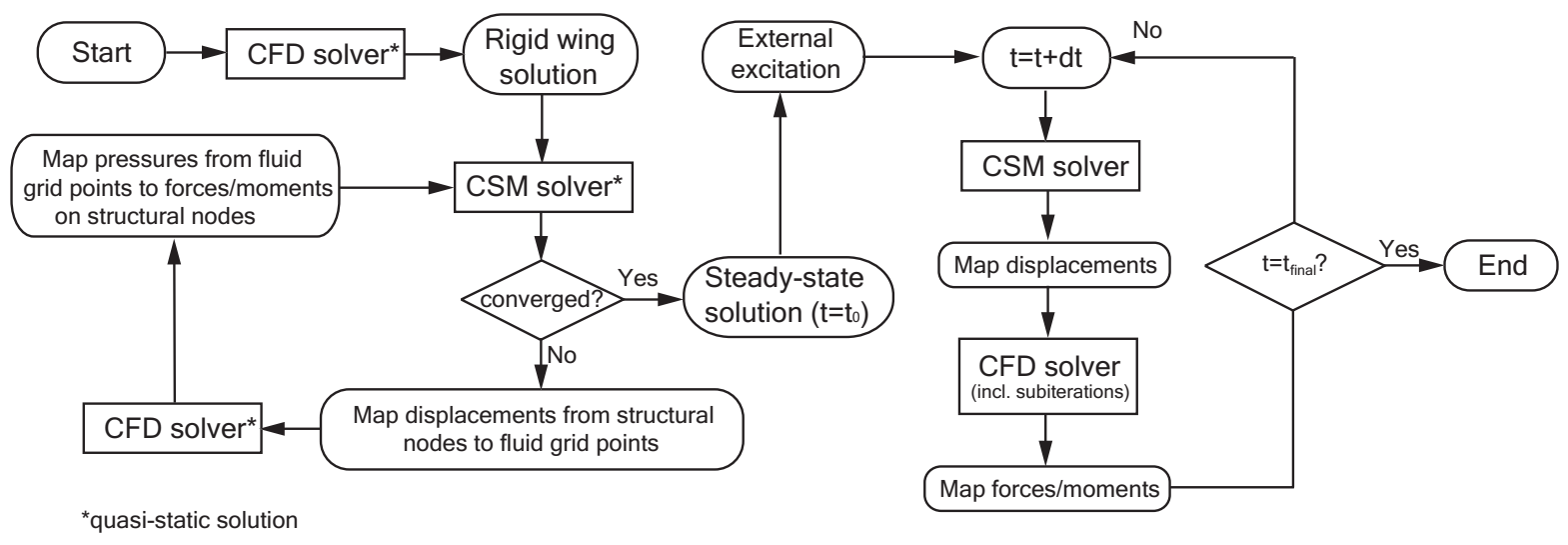

Figure 1. High-level flowchart of MDICE coupling

UM/NLABS) [23]. This combined approach recovers the actual 3-D displacements of the wetted wing surface rather than that of a beam reference line, enhancing the solid side of the fluid structure interface. The present formulation can also capture local effects in the deformation field, such as camber bending, using a Ritz-like approximation of the local warping displacement field [24], although this capability is not utilized in the current study.

\section{B. Aerodynamics}

On the fluid side, we employ AFRL's Air Vehicle Unstructured Solver (AVUS) - a finite-volume, cell centered, Euler/RANS code. Fundamentally, AVUS uses the exact Riemann solver of Gottlieb and Groth but has been modified to second order accuracy in both space and time [25]. For this work, we will primarily solve the Euler equations, and thus assume inviscid and adiabatic flow. Exceptions will be noted.

To accommodate aeroelastic applications, the CFD volume mesh is deformed within an AVUS module, using an elasticity-based PDE scheme [26] rather than a spring analogy. This method of mesh motion can account for very large mesh displacements without generating negative cell volumes, since the stiffness of mesh cells is taken as inversely proportional to their volume. Using this scheme, bending displacements approaching 50\% span have been achieved, and there is potential for still larger deformation.

\section{Fluid-Structure Interaction}

The fluid-structure interaction is managed by an interface module in MDICE. MDICE interpolates surface pressures returned from AVUS onto a fluid-structure interface: the 3-D wetted surface of the wing. Pressures are integrated to obtain resultant forces at grid points around a number of wing cross-sections (the number of cross sections corresponds to number of nodes along the spanwise discretization of the structure). These forces are then passed to the structural model. MDICE also extrapolates the displacements returned by NLABS back onto the fluid-structure interface. The interpolation algorithms are based on the work of [27,28], and ensure consistent force and conservative energy transfer at the interface.

The solution advances in time using the first-order accurate, conventional serial staggered (CSS) method [29], which is outlined in Fig. 2. In this method, both solvers are permitted to iterate independently toward convergence within a global time step, and the fluid solver may use a smaller time step and subcycling [29] to promote both convergence and stability. Solution information, in the form of pressures and displacements, is exchanged only once per global time step. Time step duration has no effect on the steady-state structural 
deformation, so large (100 s) global time steps are used to accelerate convergence. For time-accurate simulations, a much smaller time-step is required, and this is discussed later.

For the remainder of this work, the coupled FSI solver will be referred to as HiFi-VFA.

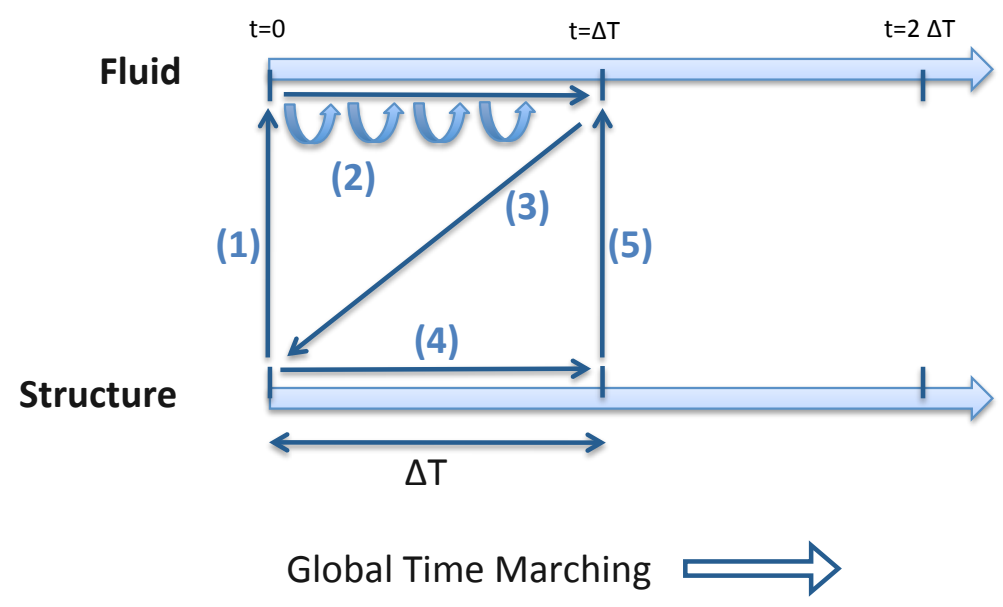

Figure 2. Conventional Serial Staggered (CSS) Coupling Procedure

\section{Flutter Prediction}

One of the primary objectives of this effort is to assess the aeroelastic stability of a very flexible aircraft. A time domain simulation is ideal for this problem, because although it is computationally intensive, the formulation can take into account aerodynamic nonlinearities and discontinuities which are not represented by the traditional frequency domain approach. McNamara and Friedmann provide an excellent review of the current state of the art in time-domain stability analysis [30].

In this work we will evaluate two approaches to determine the flutter boundary, both based on the development of an autoregressive, moving average (ARMA) model. This model is a difference equation, which, with $2 M$ Auto-Regressive (AR) and $N$ Moving Average (MA) coefficients, takes the form,

$$
y_{k}=-\sum_{i=1}^{2 M} a_{i} y_{k-i}+\sum_{i=1}^{N} b_{i} u_{k-i} .
$$

where, $y_{k}$ is a discrete time output, $u_{k}$ is a discrete time input, $M$ is the number of frequencies or modes represented by the model, the coefficients $a_{i}$ represent the aeroelastic system dynamics, and in the case of $N=1, b_{1}$ can be used to determine the system's static aeroelastic offset [31]. Given the model order along with discrete time IO data, one can determine the ARMA parameters in a linear least-squares fashion, as follows. Eq. 1 is converted into the compact form

$$
\tilde{y}=H \hat{x}+e
$$

where $\tilde{y}$ are the measured or computed output data, $H$ is a matrix of basis functions, (measured data in this case) $\hat{x}$ is a vector of estimated parameters, and $e$ represent the residual model errors. Specifically, $H$ is a $\left(N_{s}-2 M \times k\right)$ matrix, $\tilde{y}$ is a $\left(N_{s}-2 M \times 1\right)$ vector, and $\hat{x}$ is a $(k \times 1)$ vector, given as 


$$
\begin{aligned}
& H=\left[\begin{array}{cccc|cccc}
y_{2 M} & y_{2 M-1} & \cdots & y_{1} & u_{N} & u_{N-1} & \cdots & u_{1} \\
y_{2 M+1} & y_{2 M} & \cdots & y_{2} & u_{N+1} & u_{N} & \cdots & u_{2} \\
\vdots & \vdots & \ddots & \vdots & \vdots & \vdots & \ddots & \vdots \\
y_{N s-1} & y_{N s-2} & \cdots & y_{N s-2 M} & u_{N s-1} & u_{N s-2} & \cdots & u_{N s-N}
\end{array}\right] \\
& \tilde{y}=\left\{\begin{array}{c}
y_{2 M+1} \\
y_{2 M+2} \\
\vdots \\
y_{N s}
\end{array}\right\} ; \quad \hat{x}=\left\{\begin{array}{llll|llll}
-a_{1} & -a_{2} & \ldots & -a_{2 M} & b_{1} & b_{2} & \cdots & b_{N}
\end{array}\right\}^{T}
\end{aligned}
$$

By minimizing the residual $e$, we obtain the least-squares estimate of the ARMA parameters, $\hat{x}$ :

$$
\hat{x}=\left(H^{T} H\right)^{-1} H^{T} \tilde{y}
$$

Note that the order of the matrix inverse is $k \ll N_{s}$, such that the data length plays little role in the cost of the method.

Clearly, determining the optimal model order (number of AR and MA coefficients) is critical to the success of these methods. An overparameterized model (too many coefficients) may generate responses that are overly sensitive to corruption by noise, and underparameterization may produce a model with insufficient flexibility to represent the true dynamics of the system. Two popular criterion for model selection are the Akaike information criterion (AIC) and Bayesian information criterion (BIC) defined by

$$
\begin{gathered}
A I C=N_{s} \ln \left(\frac{R S S}{N_{s}}\right)+2 k+\frac{2 k(k+1)}{N_{s}-k-1} \\
B I C=N_{s} \ln \left(\frac{R S S}{N_{s}}\right)+k \ln N_{s}
\end{gathered}
$$

where $N_{S}$ is the number of observations or samples, $k$ is number of model parameters $(k=2 M+N)$, and $R S S$ is the residual sum of squares between the estimated and observed time series. BIC generally places a larger penalty on large model orders, since $\left(k \ln N_{S}>2 k\right) \forall\left(N_{S}>8\right)$. Since large order models come with increased computational cost, we will use BIC as our criterion going forward. A "better" model corresponds to a smaller BIC value, so we choose the lowest order model which has a BIC within 5\% of the minimum found. An example of a typical selection process is shown in Fig. 3, where we hold $N=1$, and vary the number of AR coefficients $2 M$. (Note: one can also vary $N$, create a three dimensional BIC surface, and select the global minimum.) By plotting the BIC vs. the number of AR coefficients for each of the models created, the optimum order is chosen. The case shown is an ARMA model, but the procedure does not change for AR, just fix $N=0$. If no minimum BIC is found, the model order is chosen subjectively based on relative performance in the flutter prediction metrics, discussed next.

\section{Modal Damping}

We first consider the ARMA flutter identification approach presented by Pak and Friedmann [31]. Setting the MA coefficients to zero (equivalently $N=0$ ), the system can be expressed in state-space form

$$
X_{p}^{k+1}=A_{p} X_{p}^{k} \quad y_{k}=C_{p} X_{p}^{k}
$$

where 


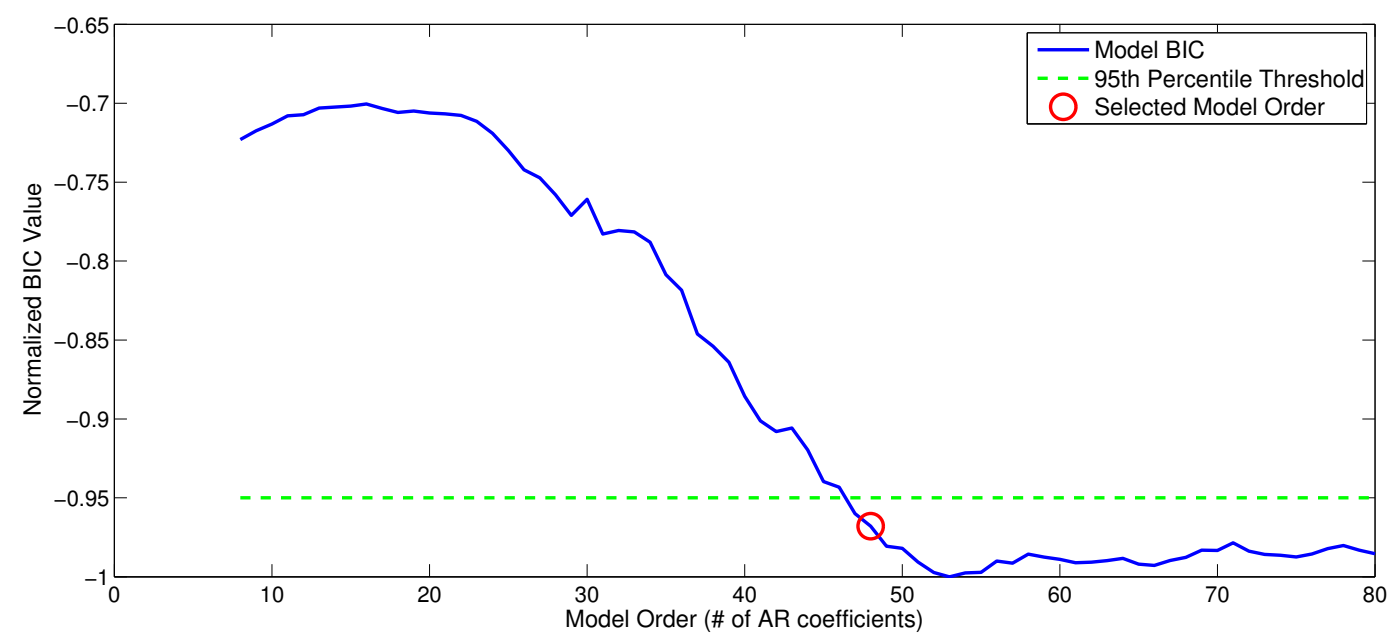

Figure 3. Example of BIC selection process

$$
A_{p}=\left[\begin{array}{ccccc}
-a_{1} & 1 & 0 & \cdots & 0 \\
-a_{2} & 0 & 1 & \cdots & 0 \\
\vdots & \vdots & \vdots & \ddots & \vdots \\
-a_{2 M-1} & 0 & 0 & \cdots & 1 \\
-a_{2 M} & 0 & 0 & \cdots & 0
\end{array}\right]
$$

and

$$
C_{p}=\left\{\begin{array}{lllll}
1 & 0 & 0 & \cdots & 0
\end{array}\right\}
$$

Here, the state vector $X_{p}^{k}$ is

$$
X_{p}^{k}=\left\{\begin{array}{c}
y^{k} \\
h_{1}(k) \\
\vdots \\
h_{2 M-1}(k)
\end{array}\right\}
$$

with entries

$$
\begin{aligned}
y_{k} & =-a_{1} y_{k-1}+h_{1}(k-1) \\
h_{1}(k) & =-a_{2} y_{k-1}+h_{2}(k-1) \\
& \vdots \\
h_{2 M-2}(k) & =-a_{2 M-1} y_{k-1}+h_{2 M-1}(k-1) \\
h_{2 M-1}(k) & =-a_{2 M} y_{k-1}
\end{aligned}
$$

\section{6 of 22}


The state space representation given in Eq. 8 is in observer form and is completely observable. Modal frequencies and modal damping can be found in the eigenvalues of the estimated matrix $A_{p}$ in Eq. 9, given as

$$
\Lambda_{j}=r_{j}+i s_{j} \text { and } \Lambda_{j+M}=r_{j}-i s_{j} \quad \text { for } j=1,2, \ldots, M
$$

Since ARMA development occurs in the discrete time domain, the corresponding modal frequency and damping in continuous time are given by [31]:

$$
\begin{array}{r}
\sigma_{j}=\frac{1}{2 T_{e}} \ln \left(r_{j}^{2}+s_{j}^{2}\right)=\zeta_{j} \omega_{j} \\
\omega_{d j}=\frac{1}{T_{e}} \tan ^{-1} \frac{s_{j}}{r_{j}}
\end{array}
$$

where $T_{e}$ is the sampling period, $\zeta_{j}$ are the modal damping coefficients, and $\omega_{d j}$ are the corresponding damped frequencies. Flutter occurs when any $\sigma_{j}=\zeta_{j} \omega_{j}<0$. In practice, Pak and Friedmann assume a single MA coefficient and fictitious system input $\delta_{k}=1$, such that

$$
y_{k}=\sum_{i=1}^{2 M}-a_{i} y_{k-i}+b_{1} \delta_{k}
$$

and we will consider this approach, as well as purely autoregressive models in this work. Since modal damping may vary nonlinearly with dynamic pressure, it is best to extrapolate from the two data points closest to flutter, or if computational data exists beyond flutter, we interpolate between it and the closest subcritical data to find the flutter point.

\section{Multi-Modal FMDS}

Next we approach the problem of flutter boundary identification from within the discrete time domain with an extension of Jury's Stability criterion, originally developed by Torii and Matsuzaki [32] and later extended to multimodal systems [33]. The parameter for flutter margin for discrete time systems (FMDS) has been shown to vary linearly with dynamic pressure in both subsonic and supersonic flow regimes.

The AR coefficients from Eq. 1 can also represent the characteristic polynomial in the discrete time domain as:

$$
G(z)=z^{2 M}+a_{1} z^{2 M-1}+\cdots+a_{2 M-1} z+a_{2 M}
$$

Torii et. al [32,33] rewrite Eq. 16 as

$$
G(z)=A_{2 M} z^{2 M}+A_{2 M-1} z^{2 M-1}+\cdots+A_{1} z+A_{0}
$$

where

$$
\left\{\begin{array}{c}
A_{2 M} \\
A_{2 M-1} \\
\vdots \\
A_{0}
\end{array}\right\}=\left\{\begin{array}{c}
1 \\
a_{1} \\
\vdots \\
a_{2 M}
\end{array}\right\}
$$

The modified FMDS flutter parameter proposed is then 


$$
F_{N}=\frac{F^{-}(2 M-1)}{F^{-}(2 M-2)^{2}}
$$

where

$$
F^{-}(j)=\operatorname{det}\left(X_{j}-Y_{j}\right)
$$

and

$$
X_{j}=\left[\begin{array}{ccc}
A_{2 M} & \cdots & A_{2 M-j+1} \\
0 & \ddots & \vdots \\
0 & 0 & A_{2 M}
\end{array}\right], \quad Y_{j}=\left[\begin{array}{ccc}
A_{j-1} & \cdots & A_{0} \\
\vdots & \ddots & 0 \\
A_{0} & 0 & 0
\end{array}\right]
$$

Instability occurs when $F_{N}<0$. We should note that $F^{-}(2 M-1)>0$ is also a required condition for stability, but the quantity $F^{-}(2 M-1)$ typically exhibits nonlinear behavior with respect to dynamic pressure, which reduces its usefulness for predicting flutter.

\section{Numerical Results}

To demonstrate the current framework, we perform a variety of numerical simulations. In 2001, Smith et. al [18] coupled an structured Euler solver with a geometrically nonlinear beam solver to analyze the static aeroelastic properties of a hypothetical HALE wing. The geometric properties are reproduced in Table 1, and the structural properties and flight conditions tested are given in Table 2. The structure side of the interface grid (from NLABS) and CFD mesh are shown in Fig. 4, and the basic discretization summarized in Table 3. The surface resolution reported in Table 3 is for each surface, upper and lower. Note that this mesh lacks adequate resolution in the boundary layer for accurate viscous computations, but several viscous results are included below, only to show the trends induced by accounting for viscosity. Due to the 2D nature of the problem, the spanwise CFD resolution is low, but cell spacing is tightly clustered near the wing tip.

Table 1. HALE Wing - Geometric and Mass Parameters

\begin{tabular}{ll}
\hline \hline Property & Value \\
Semi-Span & $16 \mathrm{~m}$ \\
Chord & $1 \mathrm{~m}$ \\
Angle of Attack & $2 \mathrm{deg}$ \\
Elastic Axis & $0.5 \mathrm{c}$ \\
Center of Gravity & $0.5 \mathrm{c}$ \\
Mass / Span & $0.75 \mathrm{~kg} / \mathrm{m}$ \\
Inertia about midchord & $0.1 \mathrm{~kg} \mathrm{~m}$ \\
\hline \hline
\end{tabular}

\section{A. Static Aeroelastic Verification}

To assess the performance of the coupled solver and verify the aeroelastic coupling, we ran HiFi-VFA for a static aeroelastic case with an airspeed of $25 \mathrm{~m} / \mathrm{s}$ at an altitude of $20 \mathrm{~km}$, corresponding to Case 1 in Table 2. Since no experimental data is available for this wing, another check is made using UM/NAST [11]. The 
Table 2. HALE Wing - Structure and Flight Conditions

\begin{tabular}{lccc}
\hline \hline & Case 1 - Incompressible & Case 2 - Similar & Case 3 - Practical \\
\hline Torsional $(G J)\left(\mathrm{Nm}^{2}\right)$ & $1.0 \times 10^{4}$ & $1.0 \times 10^{4}$ & $3.0 \times 10^{5}$ \\
Bending $\left(E I_{2}\right)\left(\mathrm{Nm}^{2}\right)$ & $2.0 \times 10^{4}$ & $2.0 \times 10^{4}$ & $6.0 \times 10^{5}$ \\
Edgewise $\left(E I_{3}\right)\left(\mathrm{Nm}^{2}\right)$ & $5.0 \times 10^{6}$ & $5.0 \times 10^{6}$ & $1.5 \times 10^{8}$ \\
\hline Altitude $(\mathrm{km})$ & 20 & $20^{\mathrm{a}}$ & 20 \\
Density $\left(\mathrm{kg} / \mathrm{m}^{3}\right)$ & 0.0889 & 0.0168 & 0.0889 \\
Mach Number & $0.0847(25 \mathrm{~m} / \mathrm{s})$ & 0.3 & 0.3 \\
\hline Reynolds \# & 154,550 & 153,618 & 547,401 \\
Dynamic Pressure $(\mathrm{Pa})$ & 27.4545 & 27.4545 & 344.42 \\
\hline \hline
\end{tabular}

${ }^{\text {a }}$ Unrealistic atmosphere, used purely for a numerical study.
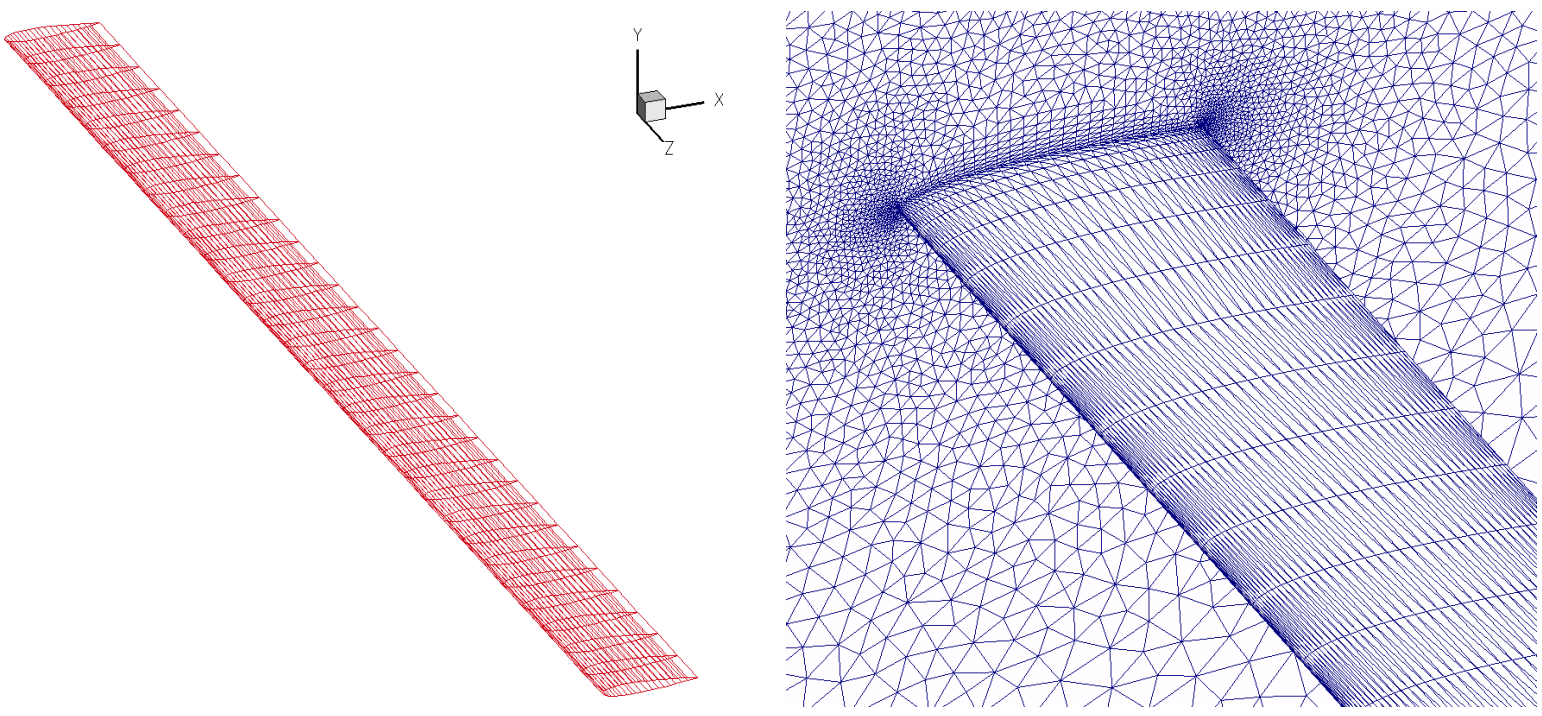

Figure 4. CSM and CFD meshes - HALE wing

spanwise flap bending and twist displacements are shown in Fig. 5. HiFi-VFA (both inviscid and viscous) agrees very well with [18]. The viscous solution slightly reduces the bending displacement, and slightly increases twist displacement.

The coupled HiFi-VFA solver shows rapid convergence to its steady state deflection (Fig. 6). A tuned parameter $\rho$ relaxes the aerodynamic loads that are applied to the structure, and facilitates nearly monotonic convergence.

Note however, the significantly larger (18\% bending, $17.4 \%$ twist) displacement predicted by UM/NAST shown in Fig. 5. This is partially a result of the two-dimensional finite-state aerodynamics formulation, which provides only an empirical 3-D lift correction at the wing tip. Another suspect is the performance of AVUS in what is essentially a zero Mach flow. To examine this possibility, fictitious flow parameters were devised (Case 2) which generate the same Reynolds number and dynamic pressure as Case 1, but at a higher Mach number -0.3 . The results of this run are shown below in Fig. 7. The minor change in the UM/NAST results is due to the small Prandtl-Glauert correction for mild compressibility at Mach 0.3 . The HiFi-VFA results however show a marked jump toward the UM/NAST results and away from reference [18]. 
Table 3. CFD/CSD Discretization - HALE Wing

\begin{tabular}{llc}
\hline \hline \multirow{2}{*}{ CFD } & Volume Cells & 642,476 \\
& Spanwise Surface Faces & 58 \\
& Chordwise Surface Faces & 86 \\
\hline \multirow{3}{*}{ CSD } & Spanwise Elements & 30 \\
& Spanwise Nodes & 31 \\
& Chordwise Nodes & 21 \\
\hline \hline
\end{tabular}
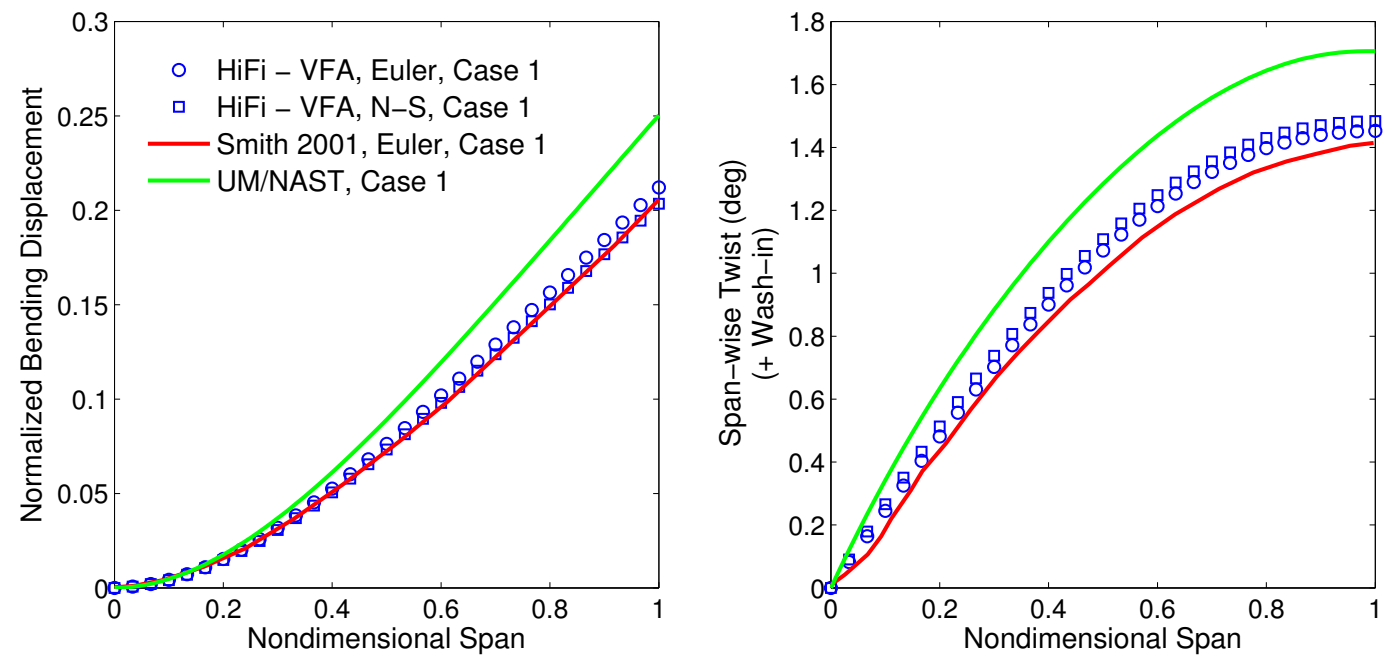

Figure 5. Case 1 - Static aeroelastic displacements
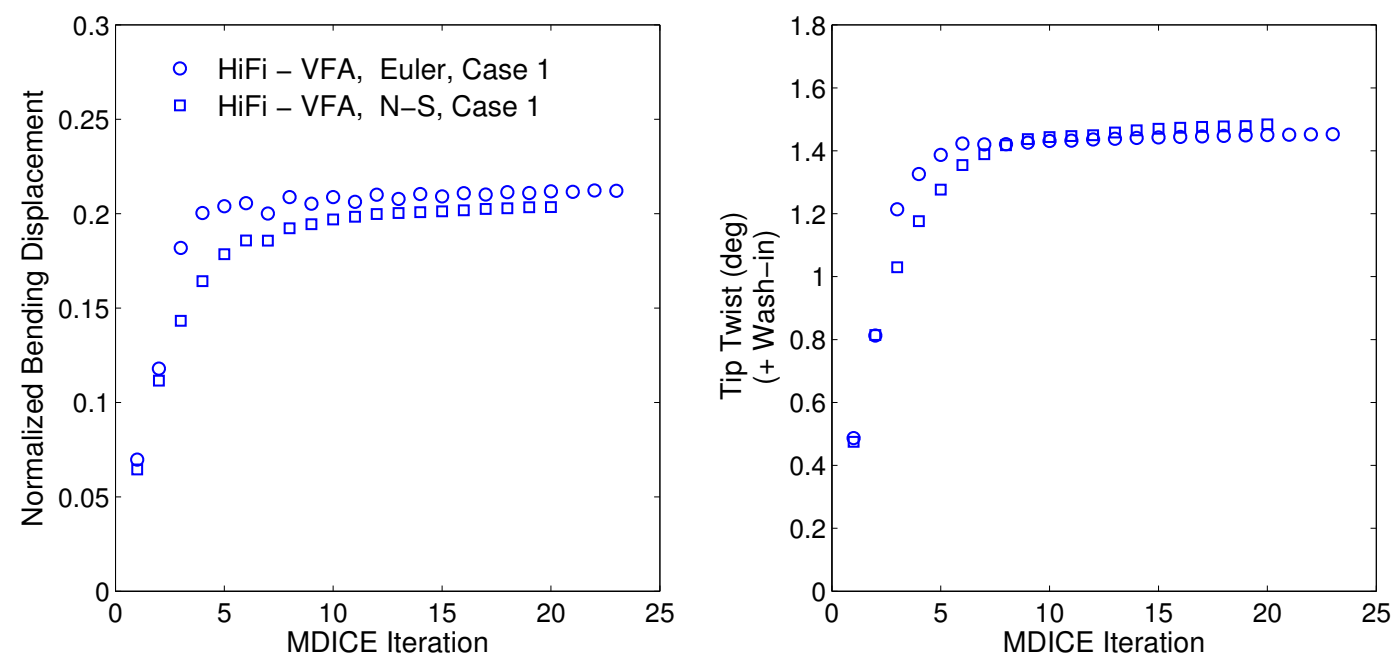

Figure 6. Case 1 - Static Convergence (Relaxation factor $\rho=0.5$ )

The relative error between UM/NAST and HiFi-VFA has been reduced to $9.4 \%$ and $8.3 \%$ for bending and twist respectively. This jump occurs when the sole difference in an otherwise similar flow is a higher Mach 
number, which suggests the current software should be used with caution for incompressible flows.
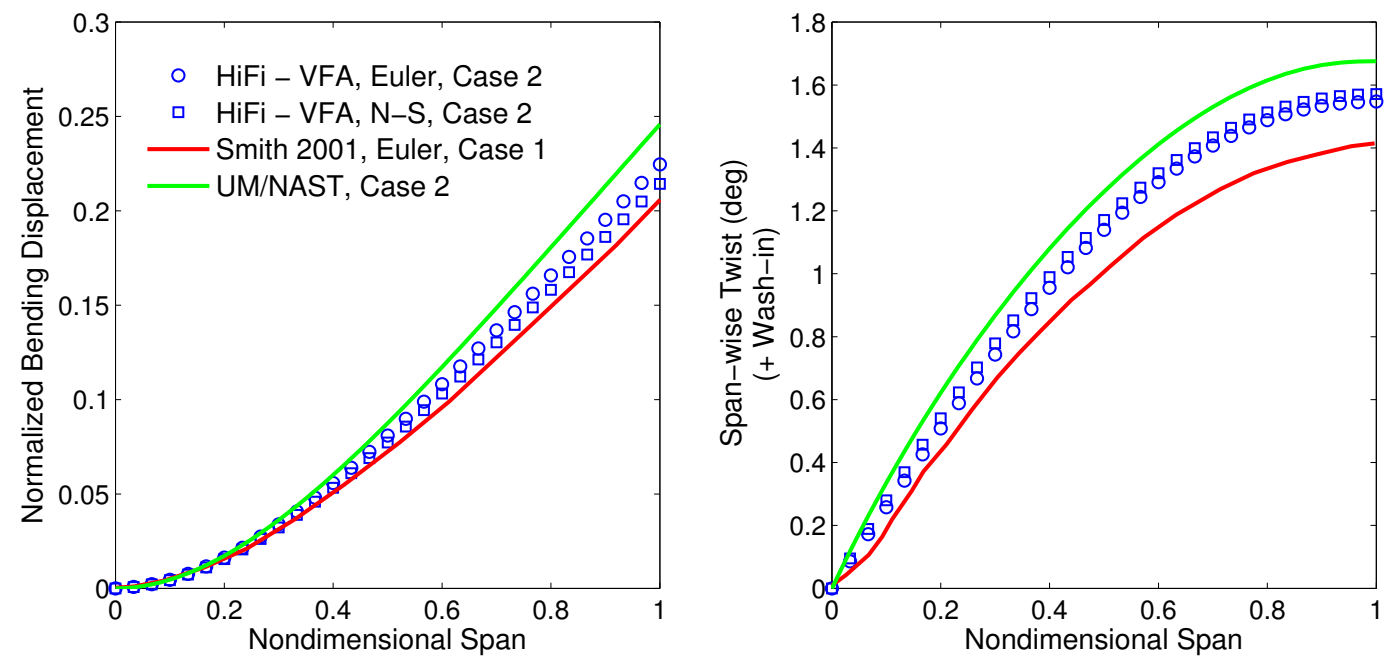

Figure 7. Case 2 - Static aeroelastic displacements

To build further confidence in the static aeroelastic coupling, Case 3 is considered. With a higher Reynolds number, and Mach number where compressibility is just beginning to play a role, Case 3 serves as the ideal verification point for the lower-order UM/NAST, and high-fidelity HiFi-VFA. As expected, the static aeroelastic displacements agree very well, and are shown in Fig. 8.
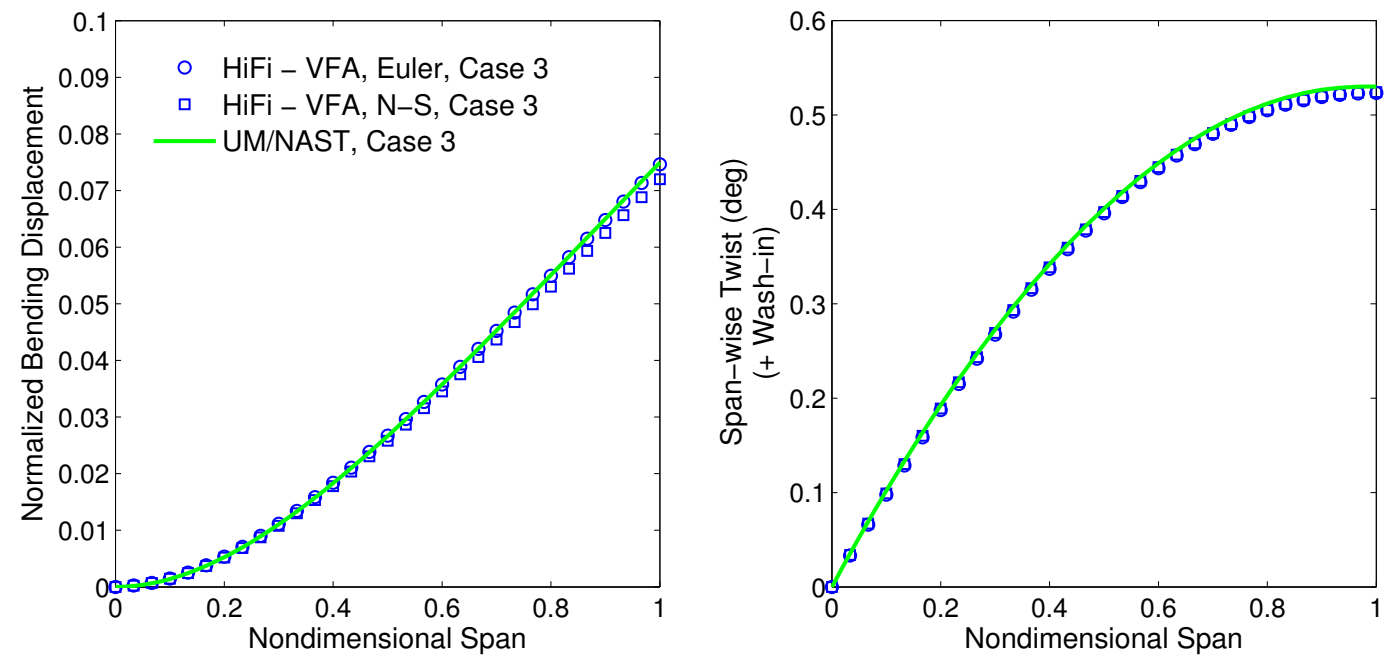

Figure 8. Case 3 - Static aeroelastic displacements

\section{B. Dynamic Aeroelasticity - Transient Response}

HiFi-VFA is loosely coupled using the CSS method, which is first order accurate in time. For flutter prediction it is critical to accurately resolve time-domain transients is both the CFD and CSM solution. To begin, the HiFi-VFA solver is run to static aeroelastic convergence at Mach=0.3. Next, the total structural state 
vector is multiplied by 1.15 , thus all translations and rotations are increased equally. The structure is then released and its aeroelastic response is recorded for 3 different time steps. A short segment of the resulting time history is shown below in Fig. 9. One can see that the transients are adequately resolved using a time step of $0.001 \mathrm{~s}$.
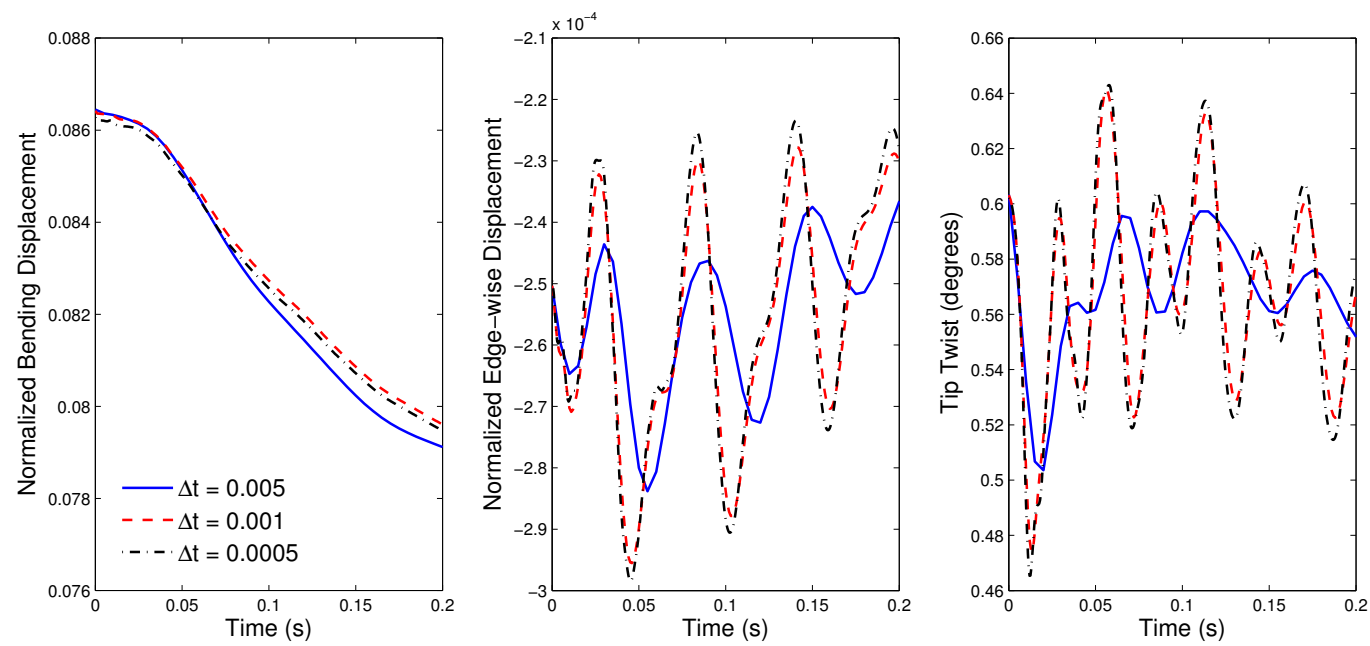

Figure 9. Case 3 - HiFi-VFA Global time step study - equal time step in each partition

Since the mesh motion algorithm requires about half of the computational time per time step, it is desirable to update the mesh as infrequently as possible. In other words, can we run multiple CFD time steps between every structural update (global time step), and thus minimize computational cost while still capturing the aeroelastic transients? To verify that we compared the run with global $\Delta t=0.001$ s from Fig. 9 with a run in which 10 CFD time steps were taken for every structural time step. This allows a CFD time step of 0.001 seconds, and structural (global) time step of 0.01 seconds. The result in Fig. 10 shows that in fact, this method does not capture the transients seen in the previous simulation which used identical time steps for both partitions. The required time step is very small, which shows the inherent disadvantage of first-order accurate solutions, and implementation of a second-order accurate scheme should be a priority. Nevertheless, accurate aeroelastic solutions can be performed given the appropriate temporal resolution.

To again build confidence in the quality of these dynamic simulations, we compare HiFi-VFA and UM/NAST, this time using an excitation similar to a backward step. To begin, the HiFi-VFA solver is run to static aeroelastic convergence at a Mach number which gives a static displacement about $15 \%$ larger than $\mathrm{M}=0.3$. Next, the Mach number is stepped down to 0.3, and the CFD solution is allowed to converge in the steady-state sense to this new mesh deflection. Lastly, the structure is released, and the time-accurate aeroelastic response is recorded. The same upper Mach number $(\mathrm{M}=0.3165)$ is used for both $\mathrm{UM} / \mathrm{NAST}$ and HiFi-VFA. Figure 11 indicates that the dynamics of the aeroelastic system are essentially the same in both codes. The frequency content of the two computed time histories is nearly identical, and the responses differ only by a steady-state offset. This offset can be attributed again to the 2D nature of the UM/NAST solution, and to the difficulty Euler codes have in accurately predicting drag. It is interesting to note (in this result and those that follow) the strong interaction between the chordwise bending and torsional degrees of freedom [5]. 

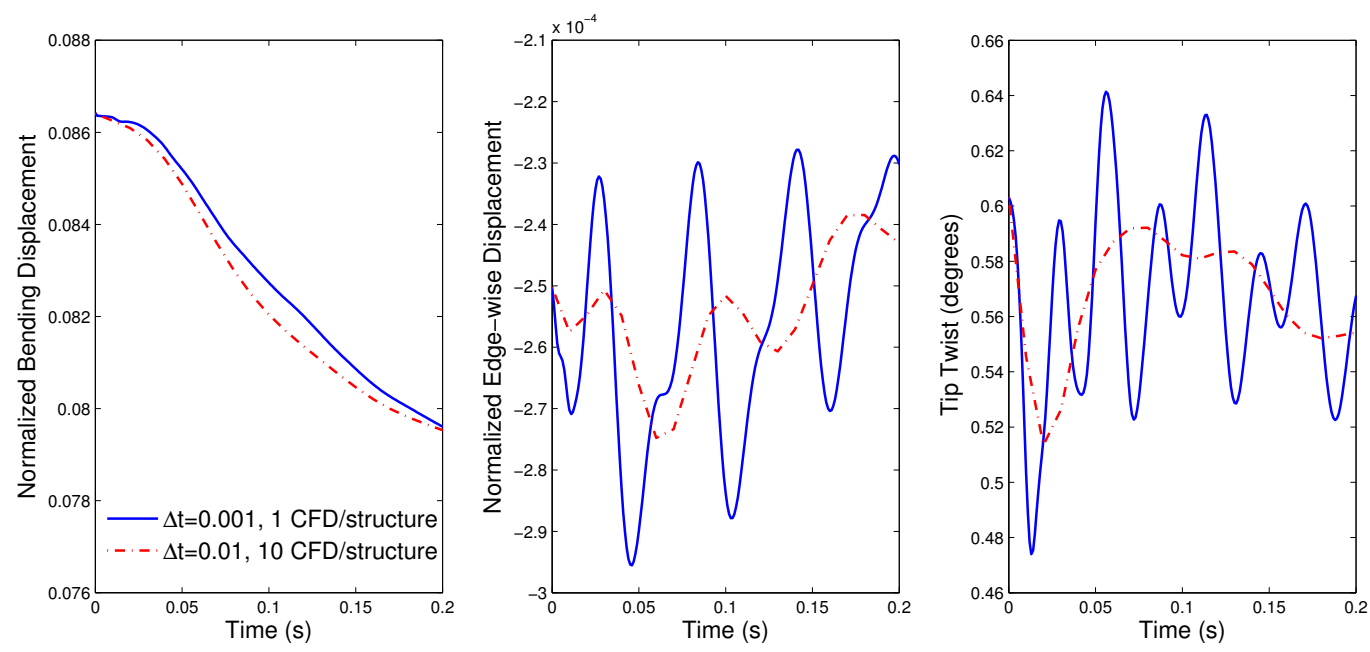

Figure 10. Case 3 - HiFi-VFA Global time step study - unequal time steps
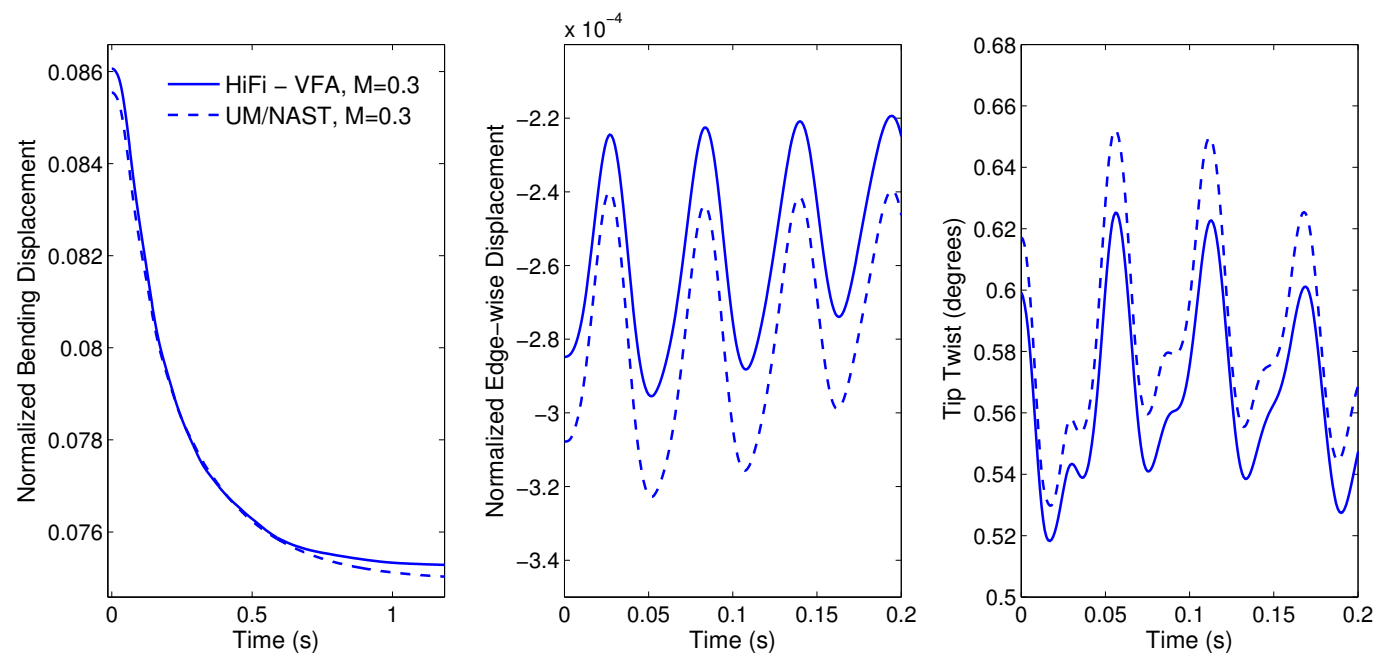

Figure 11. Case 3 - HiFi-VFA vs. NAST aeroelastic dynamics

\section{Aeroelastic Stability}

To examine aeroelastic stability in the time domain, we must perturb the system and observe the aeroelastic response, looking for one of four things: (1) damped oscillation (stable), (2) oscillatory growth without bounds (flutter), (3) oscillatory growth to limit cycle oscillation (LCO), or (4) non-oscillatory growth without bounds (divergence). In a short time simulation, we don't expect to see the development of an LCO, and the aircraft's divergence speed is likely beyond its flutter speed, so by increasing dynamic pressure from below, we minimize the possibility of number 4 . We can however, monitor damped oscillation, and (in the world of computation), safely observe unbounded growth. Using this data we predict the aircraft's stability boundary.

\section{Excitation}

The wing perturbation in this study can take one of two forms: (a) a backward step in flow speed, and (b) a sinusoidal force. Nondimensional illustrations of these inputs (Fig. 12), and their corresponding transient 
responses (Fig. 13) are given for two Mach numbers: $\mathrm{M}=0.3, \mathrm{M}=0.45$.

Stepping the flow speed promotes the richer transient response in terms of frequency content, but comes with two drawbacks: (1) adjusting the flow is not a direct input to the structure in the sense that a transfer function is logical, and (2) for the majority of the captured response, the system is non-stationary. We mitigated the latter by taking samples near the end of the observed time history, which vary much more slowly than the initial transients. The backward velocity step is defined as

$$
U_{\infty}= \begin{cases}U_{h} & t<0 \\ U_{i} & t \geq 0\end{cases}
$$

where $U_{h}$ is a heightened flow speed responsible for the initial increased deflection, and $U_{i}$ is the flow speed where the response is recorded.

To generate a stationary perturbation around the steady state aeroelastic equilibrium, a single-period sinusoidal force is applied to the wing tip in the chord-wise direction. The magnitude of the force $(50 \mathrm{~N})$ is held constant for every flight condition, but the frequency is scaled to the approximate damped chordwise bending natural frequencies $\left(\omega_{d_{i}}\right.$, Table 4$)$ observed using the backward step method. The values are approximate because the time histories allowed an FFT resolution of about $0.5 \mathrm{~Hz}$. However, exact values are unnecessary in this case because the response magnitude is large even with these approximations, and the aerodynamic damping in UM/NAST is slightly lower such that the damped natural frequencies do not match precisely between codes. The excitation is given as

$$
F_{\text {sine }_{i}}= \begin{cases}0 & t<0 \\ 50 \sin \left(\omega_{d_{i}} t\right) & 0 \leq t \leq \frac{2 \pi}{\omega_{d_{i}}} \\ 0 & t>\frac{2 \pi}{\omega_{d_{i}}}\end{cases}
$$

Table 4. Damped natural frequencies of HALE wing

\begin{tabular}{c|ccccc}
\hline \hline Mach Number & 0.30 & 0.35 & 0.40 & 0.45 & 0.50 \\
$2 \pi \omega_{d_{i}}(\mathrm{~Hz})$ & 18.75 & 15.00 & 11.50 & 8.75 & 6.25 \\
\hline \hline
\end{tabular}

\section{Flutter Prediction}

The flutter prediction process involves three steps. For each flow condition we (1) fit the ARMA model to the data, and (2) extract damping and flutter parameters. Lastly we (3) extrapolate to find the dynamic pressure at which the damping or the flutter parameter is zero. The flutter speed for the HALE wing is first calculated in UM/NAST by an eigenanalysis of the linearized system about a nonlinear steady-state deflection and is found to be $140.1 \mathrm{~m} / \mathrm{s}$. This will be considered the reference value for UM/NAST. The results of the flutter analysis, model order selected, and error with respect to this reference value are collected in Table 5. Immediately we see that a good agreement between UM/NAST's frequency domain stability analysis and the present time domain analysis is reached. The selected models (chosen in this case for their accuracy) predict the UM/NAST flutter speed within one percent. These same models, when applied blindly to subcritical HiFi-VFA time histories predict a $2 \%-5 \%$ increase in the flutter speed, which is qualitatively confirmed by examining a post-critical response at Mach $=0.5$, shown in Fig. 14. The HiFi-VFA response (solid) is just barely unstable, but the corresponding UM/NAST response grows rapidly. 


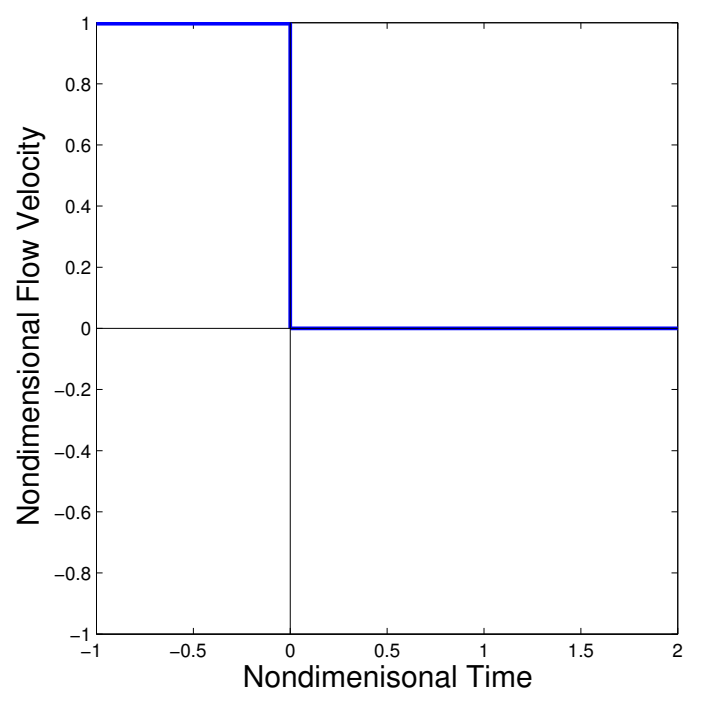

a) Backward Step

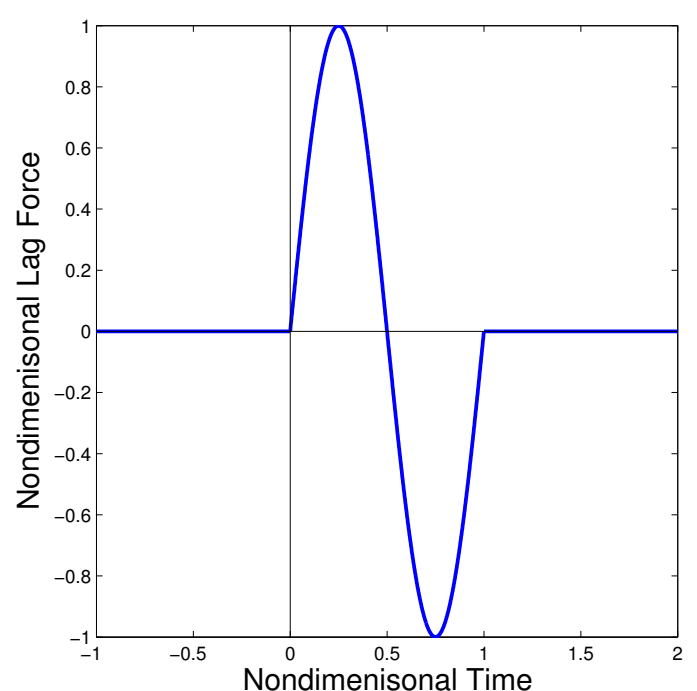

b) Sinusoidal

Figure 12. Types of excitation

Table 5. Flutter Prediction Results

\begin{tabular}{l|cc|cc}
\hline \hline Input Type & \multicolumn{2}{|c|}{ Backward Step } & \multicolumn{2}{c}{ Sinusoidal } \\
\hline Predictor & Damping & FMDS & Damping & FMDS \\
\hline Model & ARMA $(4,1)$ & ARMA(4,1) & ARMA(24,0) & ARMA $(12,1)$ \\
\hline$U_{F}[\mathrm{~m} / \mathrm{s}]$ & 140.18 & 140.09 & 140.71 & 141.00 \\
$\%$ Error & 0.06 & -0.01 & 0.43 & 0.64 \\
\hline$U_{F}[\mathrm{~m} / \mathrm{s}]$ & 146.31 & 143.62 & 142.83 & 148.18 \\
$\%$ Error & 4.18 & 2.45 & 1.91 & 5.45 \\
\hline \hline
\end{tabular}

\section{Performance of Flutter Parameters and Commentary}

Contrary to expectations, the multi-modal flutter parameter $F_{N}$ did not scale linearly with dynamic pressure, and at best appeared slightly quadratic. More commonly, $F_{N}$ exhibited a trend similar to modal damping: at first increasing with dynamic pressure, then dropping sharply on the range $0.8 q_{F}<q<q_{F}$. An example of the latter is given in Fig. 15 where the response of the backward step is modeled by an ARMA(4,1) with constant input as in [31]. Note the linear decrease in modal damping also apparent in this plot. This is neither consistent nor expected among other models, so we use the last two available sub-critical points to extrapolate modal damping in every case. Figure 16 shows a typical modal damping response, which represents reasonably mild flutter. Here $F_{N}$ is linear, as expected, on the range $0.4 q_{F}<q<q_{F}$ [33], but for the current work, this is a best case scenario - the exception rather than the rule. Often, linear extrapolation is only feasible using the last two sub-critical test points, negating any supposed advantage of the $F_{N}$ parameter over modal damping. Moreover, the cases in Fig. 15 and 16 do not take into account the variability in the estimation process, discussed next.

In order to fit an ARMA model to these computational responses, it is found that we must add a small amount of noise to the observed signal - add too much and it makes the signal difficult to fit, too little and 

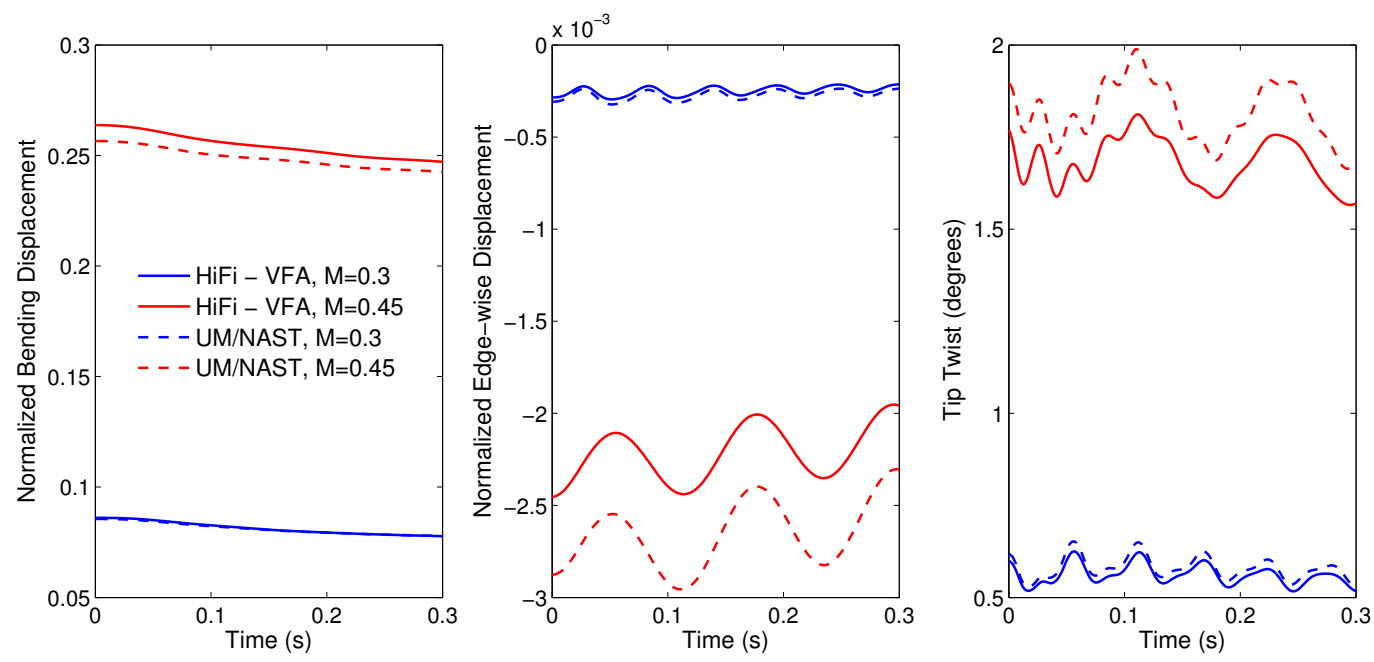

a) Backward Step
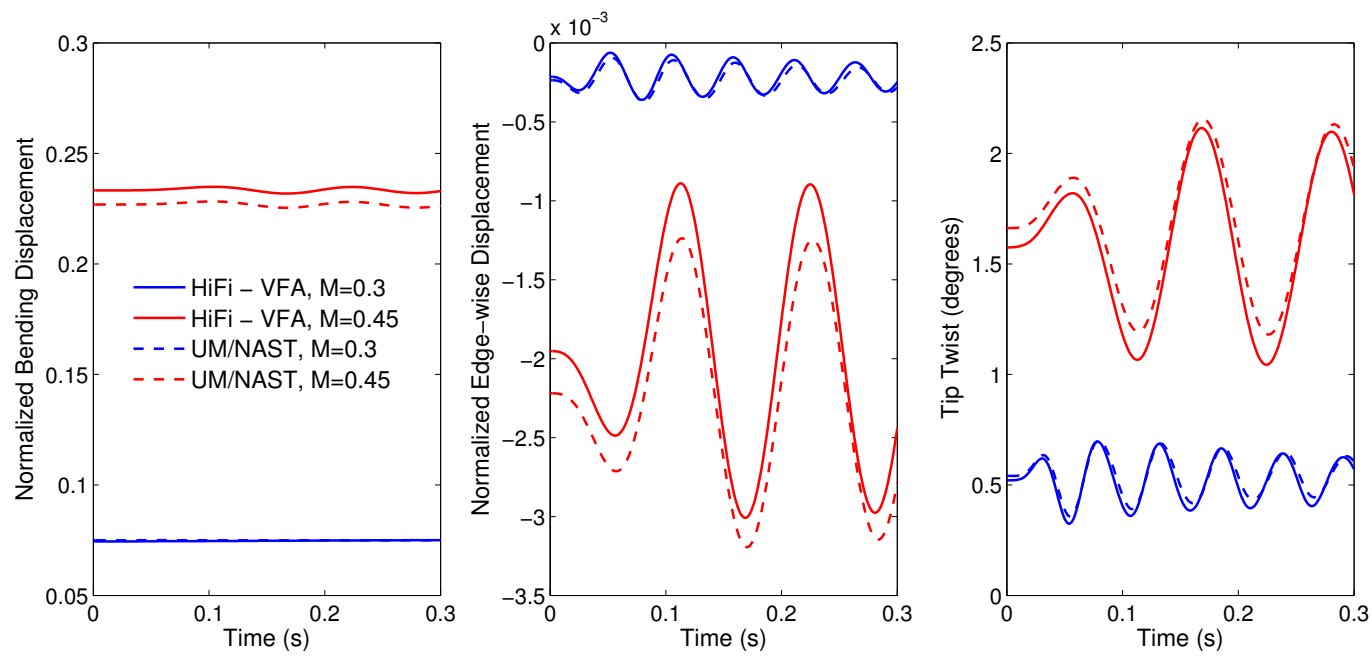

b) Sinusoidal

Figure 13. Characteristic excitation responses

the least squares algorithm is unstable. By trial, the proper magnitude of noise is found as

$$
y_{\text {noise }}=y_{0}+w g n \frac{\left\|y_{0}\right\|_{\infty}}{1000}
$$

where $w g n$ is a white gaussian noise vector equal in length to the time sampled history $y$. When creating an ARMA model, it is mildly beneficial to remove the steady state offset such that the series has a nearly zero mean. However, in the case of AR models, removing the mean is practically required. The alternative is to create extremely high-order models $(>60)$ which make flutter prediction difficult.

An additional effect of adding noise to the signal is that the flutter parameter takes on a stochastic nature. Thus each of the flutter speeds in Table 5 is the average of 100 individual predictions. For a given model order, the standard deviation of the FMDS parameter is much larger than that of modal damping, and the standard deviation of modal damping tends to be constant with respect to model order, while the spread of predicted FMDS values increases with model order. Figure 17 shows 100 iterations of the prediction process from an $\operatorname{ARMA}(12,1)$ model representing the sinusoidal excitation. The standard deviation 

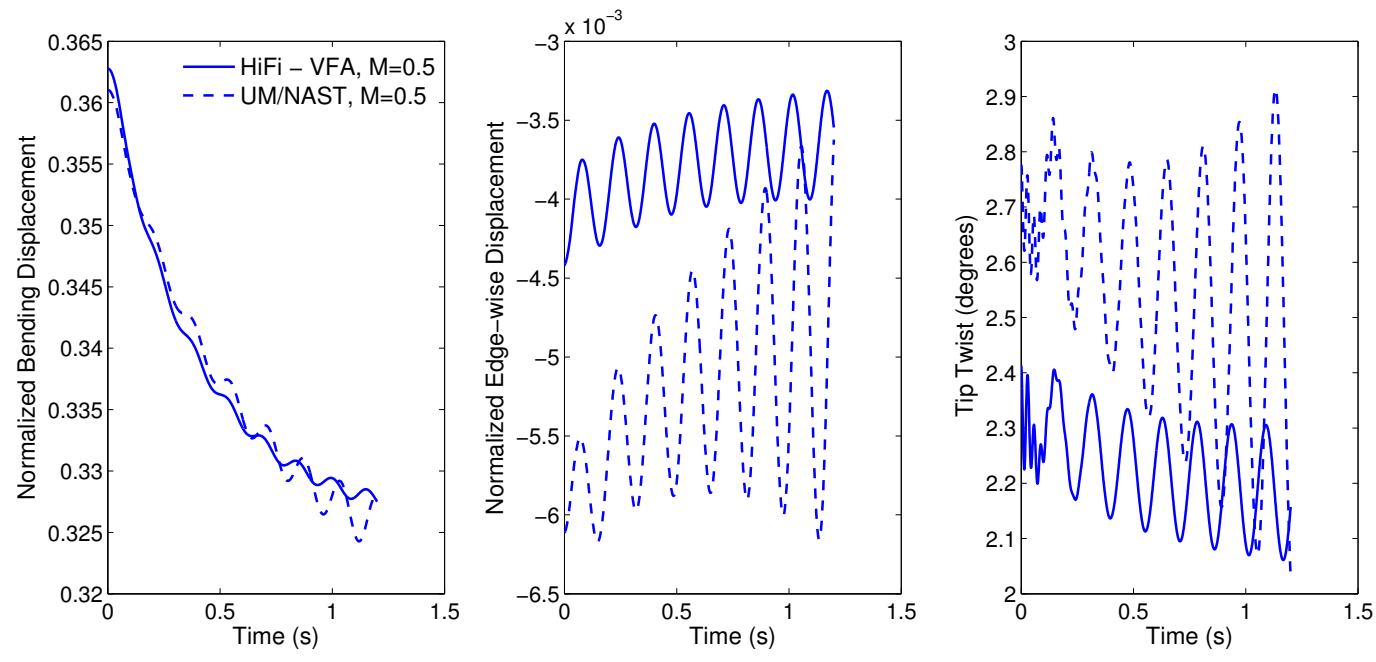

Figure 14. Postcritical response to backward step, $M=0.5$

of predicted flutter speed in this case is $0.8 \mathrm{~m} / \mathrm{s}$ and $28.5 \mathrm{~m} / \mathrm{s}$ for modal damping and FMDS respectively. Nevertheless, the average predicted value of FMDS flutter is accurate, and the computational cost for 100 averaged predictions is minimal (on the order of one minute).

\section{Computational Cost}

A word about the cost of high-fidelity simulation is in order. All of the simulations in this work were performed on an 8-core 3.2 GHz, Apple Mac Pro. AVUS and its moving mesh algorithm are parallelized and run on all 8 processors, while NLABS runs on a single core. Using this configuration, a static aeroelastic calculation requires $30 \mathrm{~min}$, and a time-accurate simulation requires approximately $10 \mathrm{hrs}$ per simulation second. The cost breakdown within a time-accurate HiFi-VFA iteration is given in Table 6.

Table 6. Cost breakdown per time-accurate iteration - HiFi-VFA

\begin{tabular}{lr}
\hline \hline Item & \% \\
\hline Mesh Motion & 56.5 \\
CFD & 40.3 \\
Interface/Overhead & 2.9 \\
Structure & 0.3 \\
\hline \hline
\end{tabular}

We see that the structural solver is relatively efficient. It is also interesting to note that the flow solution and mesh deformation consume the vast majority (96.8\%) of computational time, despite the fact that they run in parallel on 8 processors. The run times for dynamic HiFi-VFA simulations are therefore essentially inversely proportional to the amount of computational resources applied to the CFD and mesh motion modules. Considering the demonstrated scalability of these codes, and availability of modern computer clusters, large speedups are feasible. 

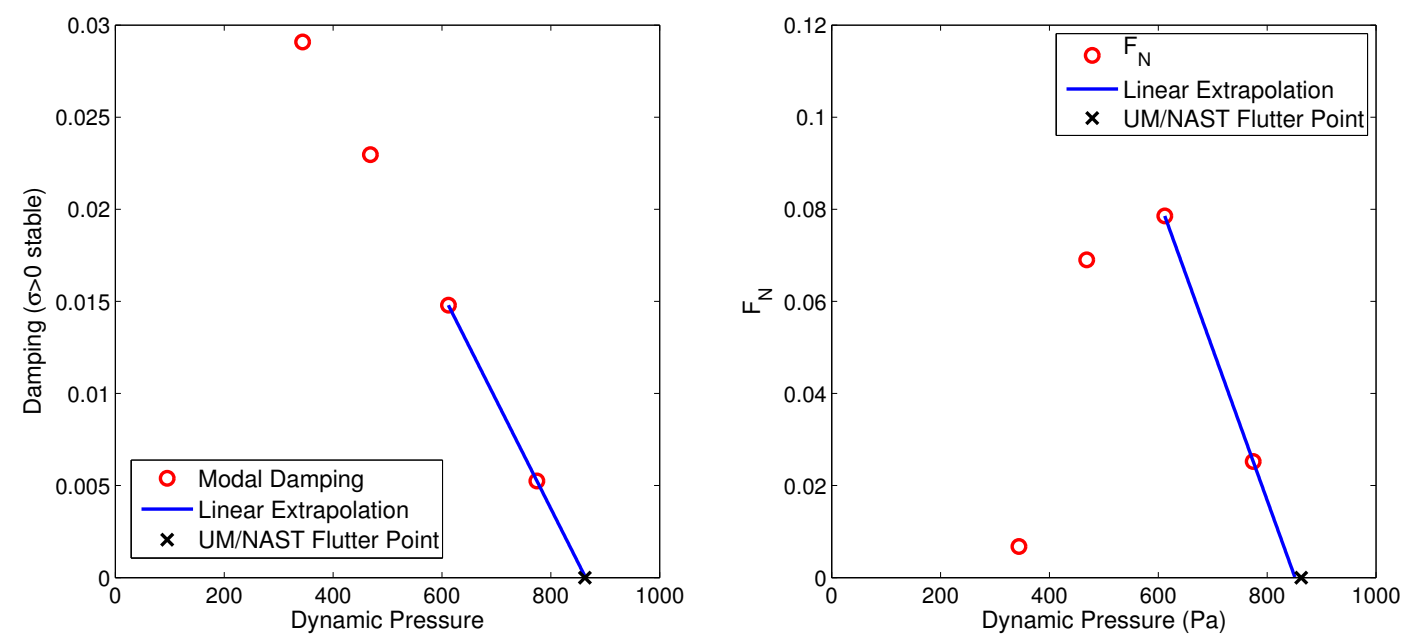

Figure 15. NAST step response, $\operatorname{ARMA}(4,1)$
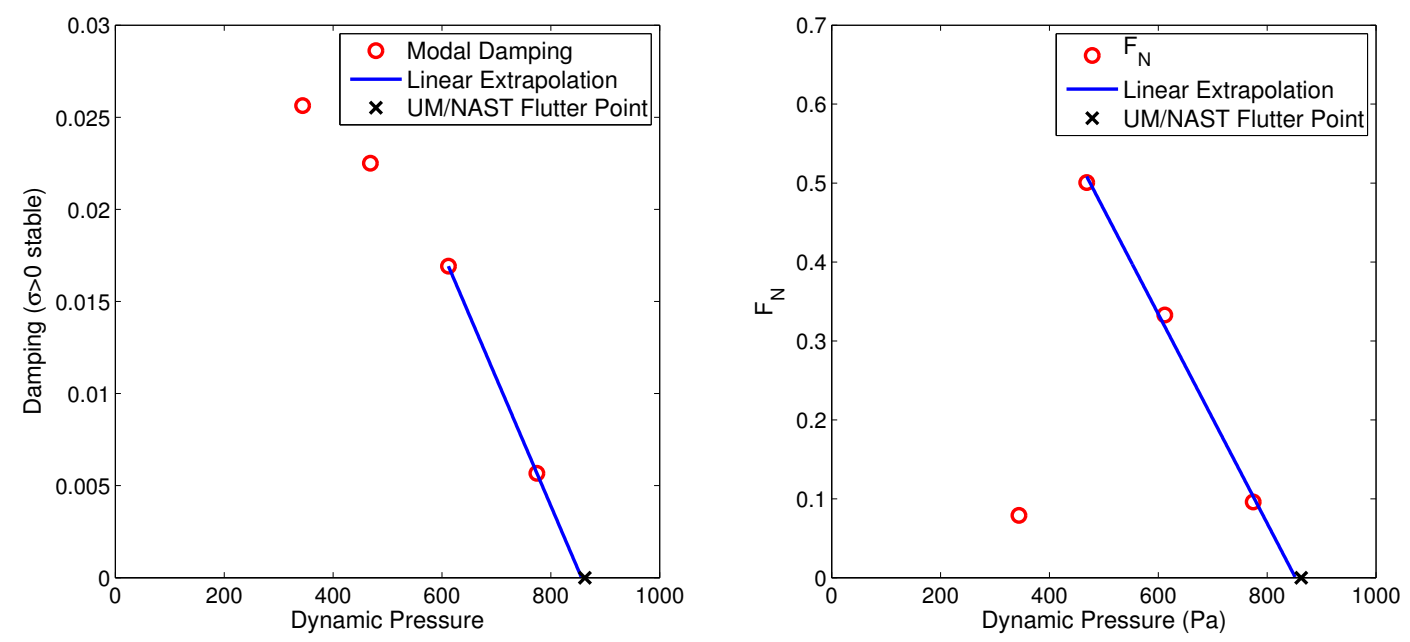

Figure 16. NAST sine response, $\operatorname{ARMA}(12,1)$ 

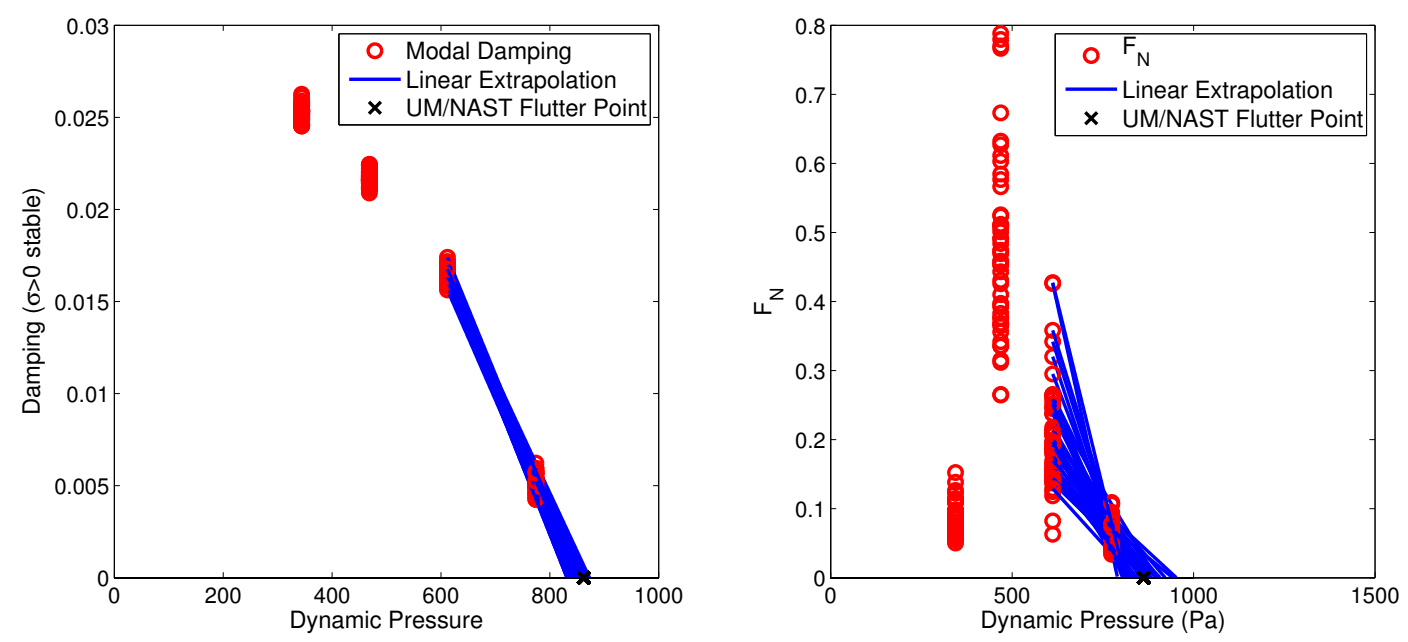

Figure 17. NAST Sinusoidal Response, $\operatorname{ARMA}(12,1), 100$ iterations

\section{Concluding Remarks / Future Direction}

The development and capabilities of a high-fidelity computational aeroelastic framework are presented. The HiFi-VFA solution is composed of a geometrically nonlinear, quasi-3D structural solver and an Euler/ Navier-Stokes flow solver. Application to a very flexible aircraft wing shows good agreement with previous computational efforts in the static aeroelastic equilibrium sense. A temporal resolution study for the first-order accurate coupling determined the necessary discretization for time accurate solutions. Verification with an in-house, lower-order aeroelasticity simulation revealed similar frequency content in dynamic aeroelastic simulations. However, these time accurate results indicate the flutter point predicted by the lower-fidelity alternative is conservative by $2-5 \%$ for this simple case.

Two flutter prediction parameters are evaluated for the HALE wing test case, which use ARMA models to fit the observed aeroelastic transients. The first, which extracts modal damping values, performed as expected: a nonlinear relation with dynamic pressure, but a good predictor of flutter when data is available close to the flutter point. This parameter tracked modal damping well using models as large as order 24. The second parameter, multi-modal FMDS $\left(F_{N}\right)$, performed with mixed results. For low order models, (essentially bi-modal) $F_{N}$ allows an accurate flutter prediction using response data as low as $q=0.5 q_{F}$, but for high order models $(>12)$, the FMDS parameter often breaks down. Both parameters are found to be sensitive to the length of data sampled, and their performance suffers for non-stationary systems.

A topic of much interest going forward is accurate simulation of HALE aircraft flight dynamics. HALE aircraft are susceptible to an aeroelastic instability called body freedom flutter, which occurs when the aircraft structural modes (generally first wing bending) are similar in frequency to, and interact with, the aircraft rigid body flight modes (generally short period pitch). These rigid body modes are clearly not accessible in the cantilevered simulation as presented, and can possess significantly lower natural frequencies than such a simulation will predict. To assess and help predict these effects, an additional MDICE module is being developed to solve the 6-DOF flight dynamics equations simultaneously. This will be discussed in a future paper. 


\section{Acknowledgments}

The authors would like to acknowledge the support of the Air Force Research Laboratory Vehicles Directorate. The technical monitor is Dr. Gregory Brooks. Additional technical communication and assistance from Dr. Edward Alyanak (AFRL), Dr. Weihua Su (UM), and Mr. Devesh Kumar (UM) is gratefully acknowledged.

\section{References}

[1] Lucia, D. J., "The SensorCraft Configurations: A Non-Linear AeroServoElastic Challenge for Aviation," Proc. 46th AIAA/ASME/ASCE/AHS/ASC Structures, Structural Dynamics and Materials Conference, AIAA Paper No. 2005-1943, Austin, TX, April 2005.

[2] Noll, T. E., Brown, J. M., Perez-Davis, M. E., and et al., "Investigation of the Helios Prototype Aircraft Mishap," Volume I, Mishap Report, January 2004.

[3] Defense Advanced Research Projects Agency, DARPA/TTO, Arlington, VA, Vulture Program: Broad Agency Announcement (BAA) Solicitation 07-15, July 2007.

[4] van Schoor, M. C. and von Flowtow, A. H., "Aeroelastic Characteristics of a Highly Flexible Aircraft," Journal of Aircraft, Vol. 27, No. 10, October 1990, pp. 901-908.

[5] Patil, M., Hodges, D., and Cesnik, C., "Nonlinear Aeroelastic Analysis of Aircraft with High-AspectRatio Wings," Proc. 39th AIAA/ASME/ASCE/AHS/ASC Structures, Structural Dynamics and Materials Conference, AIAA Paper No. 1998-1955, Long Beach, CA, April 1998.

[6] Patil, M., Hodges, D., and Cesnik, C., "Nonlinear Aeroelasticity and Flight Dynamics of High-Altitude Long-Endurance Aircraft," Proc. 40th AIAA/ASME/ASCE/AHS/ASC Structures, Structural Dynamics and Materials Conference, AIAA Paper No. 1999-1470, St. Louis, MO, April 1999.

[7] Drela, M., "Integrated Simulation Model for Preliminary Aerodynamic, Structural, and Control-Law Design of Aircraft," Proc. 40th AIAA/ASME/ASCE/AHS/ASC Structures, Structural Dynamics and Materials Conference, AIAA Paper No. 1999-1394, St. Louis, MO, April 1999.

[8] Patil, M., Hodges, D., and Cesnik, C., "Nonlinear Aeroelastic Analysis of Complete Aircraft in Subsonic Flow," Journal of Aircraft, Vol. 37, No. 5, Sept. - Oct. 2000.

[9] Patil, M. and Hodges, D., "On the Importance of Aerodynamic and Structural Geometric Nonlinearities in Aeroelastic Behavior of High-Aspect-Ratio Wings," Proc. 41 st AIAA/ASME/ASCE/AHS/ASC Structures, Structural Dynamics and Materials Conference, AIAA Paper No. 2000-1448, Atlanta, GA, April 2000.

[10] Tang, D. and Dowell, E., "Experimental and Theoretical Study on Aeroelastic Response of HighAspect-Ratio Wings," AIAA Journal, Vol. 39, No. 8, August 2001, pp. 1430-1441.

[11] Cesnik, C. and Su, W., "Nonlinear Aeroelastic Modeling and Analysis of Fully Flexible Aircraft," Proc. 46th AIAA/ASME/ASCE/AHS/ASC Structures, Structural Dynamics and Materials Conference, AIAA Paper No. 2005-2169, Austin, TX, April 2005.

[12] Su, W. and Cesnik, C., "Dynamic Response of Highly Flexible Flying Wings," Proc. 47th AIAA/ASME/ASCE/AHS/ASC Structures, Structural Dynamics and Materials Conference, AIAA Paper No. 2006-1636, Newport, RI, May 2006. 
[13] Su, W. and Cesnik, C., "Nonlinear Aeroelasticity of a Very Flexible Blended-Wing-Body Aircraft," Proc. 50th AIAA/ASME/ASCE/AHS/ASC Structures, Structural Dynamics and Materials Conference, AIAA Paper No. 2009-2402, Palm Springs, CA, May 2009.

[14] Wang, Z., Chen, P., Liu, D., and Mook, D., "Nonlinear-Aerodynamics/Nonlinear-Structure Interaction Methodology for a High-Altitude Long-Endurance Wing," Journal of Aircraft, Vol. 47, No. 2, MarchApril 2010, pp. 556-566.

[15] Peters, D. and Johnson, M., "Finite-State Airloads for Deformable Airfoils on Fixed and Rotating Wings," Symposium on Aeroelasticity and Fluid/Structure Interaction, Proc. Of the Winter Annual Meeting, ASME, Nov. 1994.

[16] Su, W. and Cesnik, C., "Nonlinear Aeroelastic Simulations of a Flapping Wing Micro Air Vehicle Using Two Unsteady Aerodynamic Formulations," Proc. 51st AIAA/ASME/ASCE/AHS/ASC Structures, Structural Dynamics and Materials Conference, AIAA Paper No. 2010-2887, Orlando, FL, April 2010.

[17] Garcia, J. A. and Guruswamy, G. P., "Aeroelastic Analysis of Transonic Wings Using Navier-Stokes Equations and a Nonlinear Beam Finite Element Model," Proc. 40th AIAA/ASME/ASCE/AHS/ASC Structures, Structural Dynamics and Materials Conference, AIAA Paper No. 1999-1215, St. Louis, MO, April 1999.

[18] Smith, M., Patil, M., and Hodges, D., "CFD-Based Analysis of Nonlinear Aeroelastic Behavior of High-Aspect Ratio Wings," Proc. 41nd AIAA/ASME/ASCE/AHS/ASC Structures, Structural Dynamics and Materials Conference, AIAA Paper No. 2001-1582, Seattle, WA, April 2001.

[19] Palacios, R. and Cesnik, C., "Static Nonlinear Aeroelasticity of Flexible Slender Wings in Compressible Flow," Proc. 46th AIAA/ASME/ASCE/AHS/ASC Structures, Structural Dynamics and Materials Conference, AIAA Paper No. 2005-1945, Austin, TX, April 2005.

[20] Garcia, J., "Numerical Investigation of Nonlinear Aeroelastic Effects on Flexible High-Aspect-Ratio Wings," Journal of Aircraft, Vol. 42, No. 4, July-August 2005, pp. 1025-1035.

[21] Seber, G. and Bendiksen, O. O., "Nonlinear Flutter Calculations Using Finite Elements in a Direct Eulerian-Lagrangian Formulation,” AIAA Journal, Vol. 46, No. 6, June 2008, pp. 1331-1341.

[22] Kingsley, G., Siegel, Jr., J., Harrand, V., Lawrence, C., and Luker, J., "Development of a MultiDisciplinary Computing Environment," Proc. 7th AIAA/USAF/NASA/ISSMO Symposium on Multidisciplinary Analysis and Optimization, AIAA Paper No. 1998-4738, St. Louis, MO, September 1998.

[23] Palacios, R. and Cesnik, C., "Structural Dynamics of Integrally-Strained Slender Wings," International Forum of Aeroelasticity and Structural Dynamics, Amsterdam, The Netherlands, June 2003.

[24] Palacios, R. and Cesnik, C., "Reduced Structural Modeling of Integrally-Strained Wings," Proc. 45th AIAA/ASME/ASCE/AHS/ASC Structures, Structural Dynamics and Materials Conference, AIAA Paper No. 2004-2038, Palm Springs, CA, April 2004.

[25] Strang, W., Tomaro, R., and Grismer, M., "The Defining Methods of Colbalt60: A Parallel, Implicit, Unstructured Euler/Navier-Stokes Flow Solver," 37th AIAA Aerospace Sciences Meeting and Exhibit, AIAA Paper No. 1999-786, Reno, NV, January 1999.

[26] Yang, Z. and Mavriplis, D., "Mesh Deforming Code For Three Dimensional Unstructured Mesh," Users Manual v2.2.

[27] Smith, M. J., Hodges, D. H., and Cesnik, C. E. S., "Evaluation of Computational Algorithms Suitable for FLuid-Structure Interactions," Journal of Aircraft, Vol. 37, No. 2, March-April 2000, pp. 282-294. 
[28] Brown, S. A., "Displacement Extrapolation for CFD and CSM Aeroelastic Analysis," AIAA Paper No. 1997-1090.

[29] Farhat, C. and Lesoinne, M., "Higher-Order Staggered and Subiteration Free Algorithms for Coupled Dynamic Aeroelasticity Problems," Proc. 36th AIAA Aerospace Sciences Meeting and Exhibit, AIAA Paper No. 1998-0516, Reno, NV, January 1998.

[30] McNamara, J. J. and Friedmann, P. P., "Flutter-Boundary Identification for Time-Domain Computational Aeroelasticity,” AIAA Journal, Vol. 45, No. 7, July 2007, pp. 1546-1555.

[31] Pak, C.-G. and Friedmann, P. P., "New Time-Domain Technique for Flutter Boundary Identification," 1992.

[32] Torii, H. and Matsuzaki, Y., "Flutter Margin Evaluation for Discrete-Time Systems," Journal of Aircraft, Vol. 38, No. 1, January-February 2001, pp. 42-47.

[33] Bae, J., Kim, J., Lee, I., Matsuzaki, Y., and Inman, D. J., "Extension of Flutter Prediction Parameter for Multimode Flutter Systems," Journal of Aircraft, Vol. 42, No. 1, January-February 2005, pp. 285-288. 